\title{
List of Corrections
}

\section{Reviewer 1}

We would like to thank the first suggestions, namely the recomputation of the geometries at the DFT/DZP level, as well as all positive comments on all paper.

\section{Reviewer 2}

Q:“ To make sure that we keep experimental and theoretical results well separated and clearly labeled, the title of the manuscript must be changed....Also the term "beta-sheet", created to describe a secondary/tertiary structure of alpha-peptides and -proteins, (as opposed to alpha-helices), should not be used to describe structures of beta-peptides?!"

A: The title has been changed to:

Theoretical study on tertiary structural elements of $\beta$-peptides: Nanotubes formed from parallel-sheet-derived assemblies of $\beta$-peptides

A: The authors agree that the term "beta-sheet" is not fully adequate to nominate sheet structures of beta-peptides. The nomenclature of beta-peptides and their possible structures is still not clearly determined and therefore can be quite confusing for even scientists familiar with the field. The authors believe that this is an unfortunate consequence of this rapidly advancing research field. Thus, the term "beta-sheet" is changed to "sheet" in the paper. Additionally, the author have realized, that these tubular forms are rather derived from sheet structures and not actually sheets, so we also made a short note for better understanding.

page 4, paragraph 4:

"We have investigated the conformational and energetic properties of multiple stranded extended sheet-derived (or in short sheet) structures formed by simple $\beta$-peptides."

Q: "The calculations include only oligomers of the two most simple beta-amino acids, beta-hGly and beta-hAla. No structures of such oligomers have so far been determined experimentally; especially as $\mathrm{H}$ (beta-hAla)nOH is expected to be rather insoluble in normal organic solvents, whatever structure it may have. Thus, I expect that it will be difficult to prove that the calculations make a reasonable structural proposal."

A: We have inserted several notes which would further underline the comment stated above:

page 4, paragraph 4:

In the present paper, we will demonstrate that the foreseen twisting, combined with the 
experimentally observed tendency that selected $\beta$-peptides have the ability to form extended sheets, appears to such an extent that it "catalyzes" the formation of a tubular network in the gas phase, resulting in nanostructures of varying sizes.

Conclusions, page 19, first parapraph:

Thus, although the investigated simple $\beta$-Ala-containing modelpeptides do not form the theoretically observed nanostructures in solution, it seems possible to apply special sidechains with appropriately chosen polarity that would interact with the outer solvent molecules and stabilize such a structure. In contrast to the above, other sidechains would selectively coordinate small compounds inside the tube.

Q: “..., but they do not explicitly give credit to the first paper, in which a short betapeptidic sheet was actually first reported...(the publication in question is ref. No. 56 on pg.6 of the manuscript...."

A: The authors thank for this useful comment, which further supports the sheet forming ability of $\beta$-peptides. The reference in question is moved to reference 16 , and detailed considerably longer:

page3, paragraph 1:

One of the earliest and most promising "spontaneously" self-assembled parallel extended sheets were constructed by Seebach et $a l^{16,17}$. These relatively short $\beta$-peptides, namely $\beta^{3}$ - and $\beta^{2,3}$-tripeptide esters form parallel extended sheets in the crystal lattice.

\section{Reviewer 3}

Q: "The authors should considerably shorten their manuscript....Some part of data could be well shifted to the Supporting Information. ... for instance the basis set dependence of the results. ... The same concerns the BSSE effects, which are relatively small. The present Supporting Information consists anyway only of the reference for the Gaussian package, which is a bit ridiculous. I could also imagine finding considerable parts of the geometry and energy tables in the Supporting Information. The tables in the text should possible be limited to the most important structures."

A: We agree with the Reviewer, thus significant amount of the Theoretical Basis, as well as Precision and Accuracy in the Results and Discussion was shifted to the Supporting Information. This includes the geometry and the basis set-dependence tables. Additionally, the remaining data and text were reformatted, to summarize the more detailed results in Supporting Information.

Thus, despite some requested insertion, the revised manuscript is more then $25 \%$ shorter now compared to the previous submitted version.

Q: "The main problem of this study is in my opinion the problem of competition of several structures of $\beta$-peptides in a special medium. Which structures will $\beta$-peptides actually form under which conditions? The main value of the work of the authors is to 
have shown that $\beta$-peptides are possibly able to form nanotubes. This could really be interesting for peptide design. It remains; of course, open, whether they will indeed do so! Therefore, it could be useful to refer stronger to $\alpha$-peptides, where the phenomenon of tube formation by several strands is a scarce event. It could be nice to have some discussion why this event is so seldom in $\alpha$-peptides or, alternatively, why are $\beta$-peptides much more able to form such structures. Are there comparable calculations for strands of $\alpha$-peptides? May be that theory predicts such tubes also there, although they are not really formed."

A: Although in theory, similar phenomenon could be observed in case of $\alpha$-peptides, the more rigid conditions of the $\alpha$-peptide backbone possibly would not allow for as small systems as two strands such a tubular structure or such diverse hydrogen bond pattern for 3-4 stranded systems. Nevertheless the authors do not intend to draw such a conclusion without factual information. Thus, only to highlight this problem a short outlook is added to page 16 .

page 16, paragraph 3:

Note, that although these tubular structures of $\beta$-peptides are quite similar to $\beta$-barrels observed in natural proteins ${ }^{11-14}$, only two strands of $\beta$-peptides are already enough to form a tubular structure, and four strands form quite stable tubes, whereas $\beta$-barrels are usually formed from at least ten or more $\alpha$-peptide strands. This phenomenon is apparently due to the local conformational flexibility of the $\beta$-peptides.

Q: "The authors confine themselves to tubes formed by up to 4 strands. This is understandable because of the considerable computational efforts. Nevertheless, some discussion could be useful on the maximum number of strands, which could be expected in such nanotubes. Are tubes with 5,6 or more strands still imaginable? Which factors determine or limit the maximum number of strands in such a nanotube?"

A: We believe that tubes with 5,6 or even more strands are stable, since tubes can be formed utilizing various hydrogen bond types, even $i+4$, or $i+5$. Nevertheless it would be quite risky to predict any stability issue, due to many unknown parameters and in the absence of factual information.

page 16 , paragraph 2:

Based on the tendency observed in the case of three- and four-stranded systems it is quite sure that five- and six-stranded systems are also stable, or perhaps even more stable than the four-stranded ones. This prediction is even more likely if one considers that more shifted hydrogen bond patterns (i.e. $i+4$ or $i+5$ ) could also appear, which could further relax a multiple stranded system. Nevertheless the stability of these large assemblies depends on many parameters, thus their relative stability compared to the three or four-stranded systems is unpredictable at the present time. Unfortunately, geometry optimization of $25-30$ or more $\beta$-amino acids in five-six different molecules at the B3LYP/6-31G(d) level of theory would require an insurmountable amount of CPU time and possibly would not really provide new concepts. 
Conclusions, page 19, paragraph 1:

Thus, to determine the stability as a function of the number of strands and to increase the interior diameter, five- six-stranded tubes should be optimized as well, nevertheless current computational methods are not sophisticated enough to carry out such computations practically in a considerable amount of time.

Q: " The authors should explicitly give the structures of the most important nanotubes... in the Supporting Information"

A: We are pleased to present the Cartesian coordinates of all the important structures: $2 \mathrm{~d}$, 3d, and 4d. See Supporting Information.

Modifications required by the Editorial Office:

Supporting Material: the pages are now numbered as S1, S2, etc. However, due to the Cartesian coordinates of the most important molecules, the number of pages increased to more than 30 .

Complete reference 37 is now given:

(37) Fülöp, F.; Martinek, T. A.; Tóth, G. K. Chemical Society Reviews, 2006, (Advance Article), DOI: 10.1039/b501173f

In order to improve understanding and provide a clear train of thought, the following minor additional changes were applied:

The levels of theories were annotated with a short symbol, such as M1a, M2b, etc. However, the investigated model peptides were nominated as $2 \mathrm{a}, 2 \mathrm{~b}, 4 \mathrm{~d}$, etc. This could be confusing, thus for a better understanding the M1a type notations of the applied methods were changed to $\mathrm{mI}$, or $\mathrm{mI} / \mathrm{B}$ (mI with BSSE), etc.

The graphical abstract is changed to a more conceptual one. 